\title{
Studies of charm production at the Serpukhov accelerator; an experimental data review.
}

\author{
L.Tikhonova \\ Institute of Nuclear Physics, Moscow State University, Moscow, Russia, Vorobievi Gori 119899 \\ email: L्arisa@npi.msu.sul
}

ABstract: Charm production studies with different detectors at Serpukhov accelerator energies are described. The $\sigma(c \bar{c})$ estimates obtained with the BEAM-DUMP and SVD experiments do not contradict each other or the perturbative QCD predictions, while BIS-2 results notably exceed the other experiment values and the theoretical expectations.

\section{Introduction}

The production of heavy flavours in hadron-hadron interactions is a very important tool for quantitative tests of QCD and for searches for new physics. Most of the data obtained at CERN and FNAL accelerators in the energy interval $200-$ $800 \mathrm{GeV}$ do not contradict the QCD predictions. On the other hand the experimental information on charm production from the Serpukhov accelerator at the energies below $100 \mathrm{GeV}$ is scarce and contradictory. Charm studies at the Serpukhov accelerator started more then 20 years ago and are continuing. Most of the data were taken with three different types of apparatus, which have different systematics. These are the series of the PROTON BEAM-DUMP experiments, the BIS-2 experiment and the SVD experiment.

The three key ingredients one needs when calculating a charm cross-section (besides having the signals) are essentially:

1. A-dependence $\left(\sigma(p A)=\sigma(p p) A^{\alpha}\right)$, since most of experiments at Serpukhov accelerator are performed using heavy nuclear targets,

2. branching ratios, because each of the experiments can detect only some specific final states,
3. model dependency, since as a rule the experiments do not cover the whole range of $x_{F}$ and $p_{\perp}$, a model dependent $x_{F}$ and $p_{\perp}$ behaviour is required to extract a cross section from the signal.

Different assumptions regarding these points can change the extracted cross-section values remarkably. Futhermore, several other factors are often necessary to correct for cuts in the data; trigger inefficiencies, dead zones in the apparatus, limited angular acceptance, etc.

\section{BEAM-DUMP Experiments}

Experiments on the total absorption by iron of the $70 \mathrm{GeV}$ proton beam with detection of prompt neutrinos or leptons from semileptonic decays of

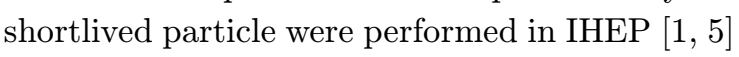
in order to estimate the charm production crosssections $\sigma(c \bar{c})$.

The first result, $\sigma(c \bar{c})=5 \pm 4 \mu b$ iij per nucleon, was obtained using a muon absorber and performed prompt electron neutrino/antineutrino detection in a neutrino detector.

An upper limit for $\sigma(c \bar{c})<4.8 \mu b$ per nucleon $(90 \%$ C.L.) [2] was obtained when the bubble chamber SCAT filled with promine freon was used as a neutrino detector. After background subtraction the total number of $\nu_{\mu}, \bar{\nu}_{\mu}$ events was found to be $5 \pm 12$, for $\nu_{e}, \bar{\nu}_{e}$ events -0.66 . 
A linear $A$-dependence taken from prompt neutrino yield calculations was assumed.

Muon yields from $p-F e$ interactions were measured in 9 sections of steel muon absorber [3] The possible contribution to the yield of muons from charm decays was evaluated. Using the Likhoded-Slabospitsky model [4], an estimate of the charm production cross section at $\sqrt{S}=11.5$ $\mathrm{GeV}$ was obtained of $\sigma(c \bar{c})=-0.3 \pm 1.2 \mu b$ per nucleon, corresponding to an upper limit of $\sigma(c \bar{c})<$ $1.6 \mu b$ at $90 \%$ C.L.

An average over four independent measurements of signals from prompt muon and electron antineutrinos $((c c)$ interactions) with the IHEPJINR neutrino detector was made in $[\underline{\underline{n}}]$. A total charm cross-section of $\sigma(c \bar{c})=0.9_{-0.9}^{+1.1} \mu b$ per nucleon was obtained using a linear $A$-dependence approximation. Two independent methods for the $\sigma(c \bar{c})$ estimation were used;

1. linear extrapolation of event numbers from an $\mathrm{Fe}$ target to an infinite density target,

2. subtraction of background from $\pi$ and $K$ decays as neutrino/antineutrino sources.

The charmed particle spectra were parametrized

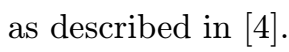

\section{BIS-2 Experiment}

The main components of the BIS-2 spectrometer are a momentum analyzing magnet, 22 planes of multiwire proportional chambers, arranged upstream and downstream of the magnet, and two multichannel threshold Cherenkov gas counters for charged particle identification. The construction of BIS-2 permits selection of charmed particles only in the regions $x_{F}>0.5$ and $p_{\perp}<1$ $\mathrm{GeV} / \mathrm{c}$. The experiment was performed in the neutron beam with an energy of $40-70 \mathrm{GeV}$ using hydrogen, carbon and aluminium targets.

The signals of charmed mesons and baryons were detected in the effective mass spectra for different hadronic decay modes $\left[\begin{array}{l}\overline{6}_{0}^{\prime} \\ \mathrm{i}\end{array}\right.$ sive production of charmed baryons $\Lambda_{c}^{+}$on carbon was observed via decays $\Lambda_{c}^{+} \rightarrow \bar{K}^{0} p \pi^{+} \pi^{-}$ and $\Lambda_{c}^{+} \rightarrow \Lambda^{0} \pi^{+} \pi^{+} \pi^{-} \quad\left[\hat{6}_{1}^{-i}\right]$. The invariant $\Lambda_{c}^{+}$ production cross section for $x_{F}>0.5, p_{\perp}<1$
$\mathrm{GeV} / \mathrm{c}$ was described using

$$
E \frac{d^{3} \sigma}{d p^{3}} \sim\left(1-x_{F}\right)^{n} e^{-b p_{\perp}}
$$

with $b=2.5 \pm 0.6(\mathrm{GeV} / \mathrm{c})^{-1}$ and $n=1.5 \pm 0.5$. The inclusive $\Lambda_{c}^{+}$production cross section per nucleon for $x_{F}>0$ was estimated to be greater than $17 \mu b$ on the assumption of an $A^{2 / 3}$ dependence for the cross section on nuclei and a constant $d \sigma / d x_{F}$ distribution.

The production of $\bar{D}$ mesons has been observed in $n p, n C$ and $n A l$ interactions. Signals for $\bar{D}^{0}$ mesons were detected in the effective mass

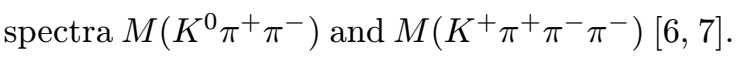
Signals for $D^{-}$mesons were detected through the $\left(K^{0} \pi^{+} \pi^{-} \pi^{-}\right)$final state. The contribution of the $K^{*+}(892)$ meson to charm meson decays was found to be substantial. The charmed meson signals in charge conjugate systems were not found.

The invariant longitudinal momentum spectra were described in terms of $\left(1-x_{F}\right)^{n}$ with $n\left(\bar{D}^{0}\right)=2.3_{-0.3}^{+0.2}$ and $n\left(D^{-}\right)=0.8 \pm 0.4$.

The transverse momentum spectra are consitent with an exponential $p_{\perp}^{2}$ behaviour having slopes of $B\left(\bar{D}^{0}\right)=1.2_{-0.9}^{+1.1}(\mathrm{GeV} / \mathrm{c})^{-2}$ and $B\left(D^{-}\right)=$ $1.8_{-1.0}^{+1.3}(\mathrm{GeV} / \mathrm{c})^{-2}$. Comparing $\Lambda_{c}^{+}$and $\bar{D}$ production in the same experiment [i], the authors conclude $\left(\Lambda_{c}^{+} \bar{D}\right)$ dominates over $(D \bar{D})$ production in the large $x_{F}$ region. The flat and similar longitudinal momentum spectra of $\Lambda_{c}^{+}$and $\bar{D}$ favour $\left(\Lambda_{c}^{+} \bar{D}\right)$ production in diffraction-like processes.

The inclusive $\bar{D}^{0}$ cross-section in $n p$ interactions at $x_{F}>0.5$ was measured to be $\sigma\left(\bar{D}^{0}\right)=$ $3.2 \pm 1.1 \mu b$, and the $A^{\alpha}$ dependence from data on $(H, C, A l)$ nuclei was described by $\alpha=0.73 \pm$ $0.23[\overline{8}]$.

The extrapolation of the $\bar{D}$ cross-section per nucleon for $x_{F}>0$ was performed with a diffractive model, which gives the lower limit $\sim 10 \mu b$, and with a $\left(1-x_{F}\right)^{n}$ parametrization, which leads to a large cross section of $20-30 \mu b$. The extrapolation to $\left(-1 \leq x_{F} \leq 1\right)[\underline{\overline{9}}]$ gives $\sigma\left(\Lambda_{c}^{+} \bar{D}\right)=$ $80 \pm 20 \mu b$.

\section{SVD Experiment}

The E-161 experiment to study charm produc- 


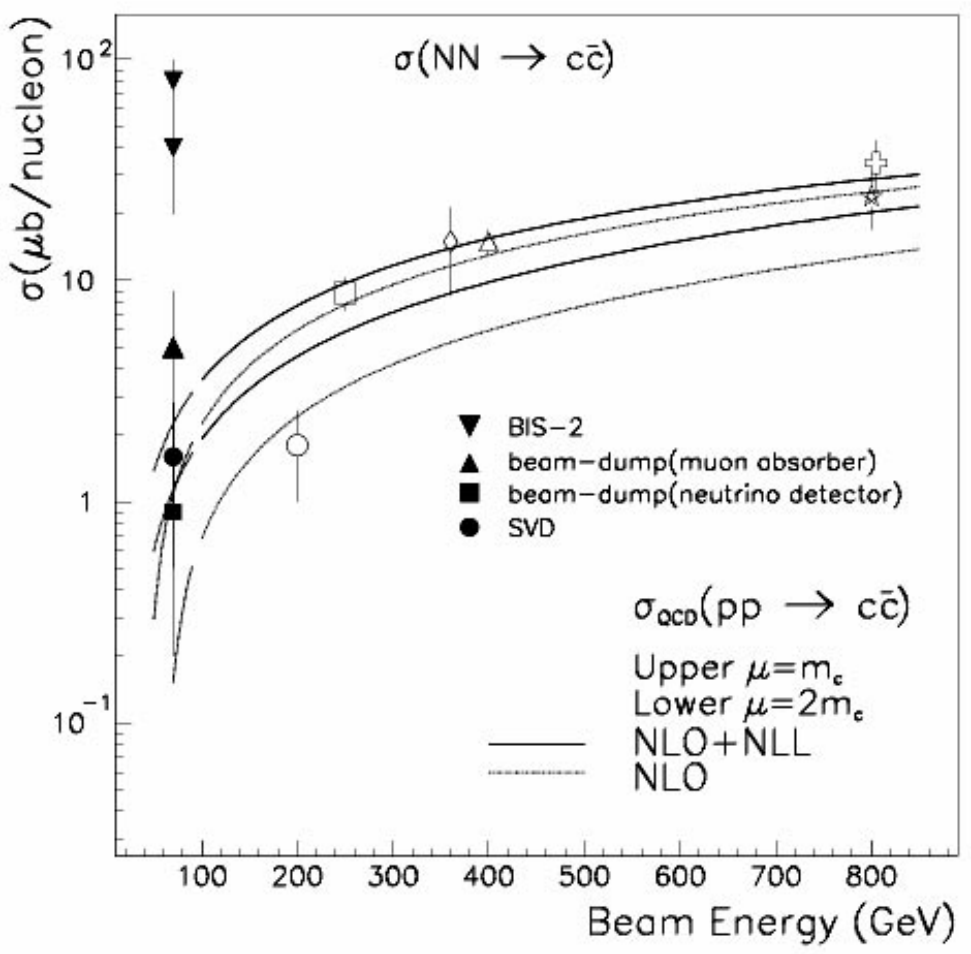

Figure 1: Total $c \bar{c}$ cross-section in nucleon-nucleon interactions as a function of beam energy. Serpukhov experimental data for the BIS-2, SVD and "beam-dump" experiments are presented. Open points correspond to CERN and FNAL data [10] for energies $250-800 \mathrm{GeV}$. The theoretical curves are taken from QCD calculations, which include soft gluon resummation [1]

tion in $p p$ interactions at $70 \mathrm{GeV} / \mathrm{c}$ was performed. This used the Spectrometer with Vertex Detector (SVD) [10 periment used the rapid cycling liquid hydrogen bubble chamber (HBC) as a precise vertex detector. A hydrogen target was used in combination with a wide aperture magnetic spectrometer with multiwire proportional chambers (31 planes).

The HBC permits measurements of the primary interaction vertex and decay points of unstable particles with a high precision of $(\triangle x=$ $\triangle y \simeq 10 \mu m, \triangle z \simeq 50 \mu m$ ) The four metre long magnetic spectrometer gives a high efficiency for detection of charged particles. The total efficiency for pion detection in the spectrometer for $\left(-1 \leq x_{F} \leq 1\right)$ is equal to $0.66 \pm 0.05$. The experimental effective mass resolutions for strange particles are $1.92 \mathrm{MeV}$ for $K_{S}^{0}$ and $2.25 \mathrm{MeV}$ for $\Lambda^{0}$. About 140 thousand $p p$ interactions were seen in the HBC fiducial volume, corresponding to a sensitivity for the experiment of 5 event $/ \mu b$.
The main background source in events with charm candidates are strange particle decays. The use of special cuts on total and transverse decay length suppresses this background significantly.

After analysis of combined bubble chamber and spectrometer information, 3 three prong (C3) decays of charged particles and 2 two prong (V2) decays of neutral particles were chosen as charmed meson candidates.

An estimate of the total charm production cross-section $\sigma(c \bar{c})$ was obtained from comparison of the experimental data and the Monte Carlo simulation using PYTHIA Version 5.702. Taking into account the different decay modes and the "charm box" dimensions, the expected numbers of C3 and V2 charm events from the processes of charm quark pair production by quark and gluon fusion in $p p$ reactions were estimated to a statistical level of 10 event $/ \mu b$, assuming $\sigma(c \bar{c})=1.07$ $\mu b$. The experimental numbers of $\mathrm{C} 3$ and V2 charm decays were corrected for the scan and 
selection efficiencies and the spectrometer accep- References

tance.

[1] A.E.Asratyan et al., iPhys. Lett. B $\mathbf{7 9}(1978)$

- After normalization to the experimentalstatis- - . 49 r

tics, a value of $\sigma(c \bar{c})=\left[1.6_{-0.7}^{+1.1}(\right.$ stat. $) \pm 0.3($ syst. $\left.)\right]$

$\mu b$ was obtained. The systematic error was mainly due to the unknown contributions from $(D \bar{D})$ and $\left(D \Lambda_{c}^{+}\right)$channels, for which the ratio was varied between 1 and 0.2 .

The mean longitudinal and transverse momentum values of observed $D$ mesons were estimated as $\left\langle\left|x_{F}\right|>=0.20 \pm 0.07\right.$ and $\left\langle p_{\perp}\right\rangle=$ $0.53 \pm 0.13 \mathrm{GeV} / \mathrm{c}$, leading to a value of $n=3.0 \pm$ 1.3 , using the $d \sigma / d x_{F}=\left(1-\left|x_{F}\right|\right)^{n}$ parametrization.

All of the mentioned $\sigma(c \bar{c})$ results are shown in figure 1, where the QCD predictions [i] 1 in, including soft gluon resummation at the next to leading-logarithmic level $[\mathrm{NLO}+\mathrm{NLL}]$, are also presented. When the resummed partonic crosssections are convoluted with hadronic parton densities, a significant improvement is found in the stability of the results with respect to changes of the renormalization scale and an increase in the mean value of $\sigma(c \bar{c})$ near threshold of approxi-

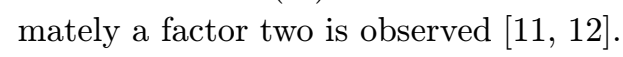

As can be seen from figure 1, the Serpukhov $\sigma(c \bar{c})$ experimental data at $\sqrt{S} \sim 10 \mathrm{GeV}$ can be divided into two groups; the first is concentrated near $\sigma(c \bar{c}) \sim(1 \div 5) \mu b$ in agreement with the latest QCD threshold predictions [11 $\overline{1}_{1}^{1}, \overline{1}, \overline{1} \bar{l}$ li and the second, obtained by extrapolation of BIS-2 results, is situated near $\sigma(c \bar{c}) \sim(40 \div 80) \mu b$. The BIS-2 estimates for $\left(-1 \leq x_{F} \leq 1\right)$ are anomalously large compared with both the theoretically expected and experimentally measured values, even those at higher energies.

New precision $\sigma(c \bar{c})$ experimental data over the whole $x_{F}$ region will be neccesary to clarify the situation. One such attempt is the upgrade of the SVD experiment to include a new active high resolution vertex detector with microstrip planes instead of the bubble chamber, a detector of gamma quanta and a multicell threshold Cherenkov counter [1] $3_{3}^{1}$, in order to obtain $10^{3} \div 10^{4}$ charm events in two years.

[2] V.V.Ammosov et al., Russ. Journ. of Nucl. Phys. 53 (1991) 999.

[3] S.V.Belikov et al., Russ. Journ. of Nucl. Phys. 58 (1995) 1993.

[4] A.K.Likhoded, S.P. Slabospitsky. Russ. Journ. of Nucl. Phys. 33 (1981) 832.

[5] S.A.Bunyatov, Yu.A.Nefedov. Russ. Journ. of Nucl. Phys. 60 (1997) 1045.

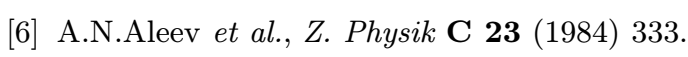

[7] A.N.Aleev et al.,

[8] A.N.Aleev et al., Russ. Journ. of Nucl. Phys. 56 (1993) 147.

[9] Yu.M.Sviridov. Russ. Journ. of Nucl. Phys. 49 (1989) 172.

[10] E.Ardashev et al., preprint INP MSU 97-8/459, Moscow (1997). E.Ardashev et al., preprint INP MSU 99-27/585, Moscow (1999).

[11] R.Bonchiani, S.Catani, M.L.Mangano, P.Nason. Nucl. Phys. B $\mathbf{5 2 9} \mathbf{9}(1998)$ 424u.

[12] M.Beneke, preprint CERN-TH/98-202 (1998). M.Beneke, V.A.Smirnov, preprint CERNTH/97-315 (1997).

[13] E.Ardashev et al., preprint IHEP 96-98, Protvino (1998). 